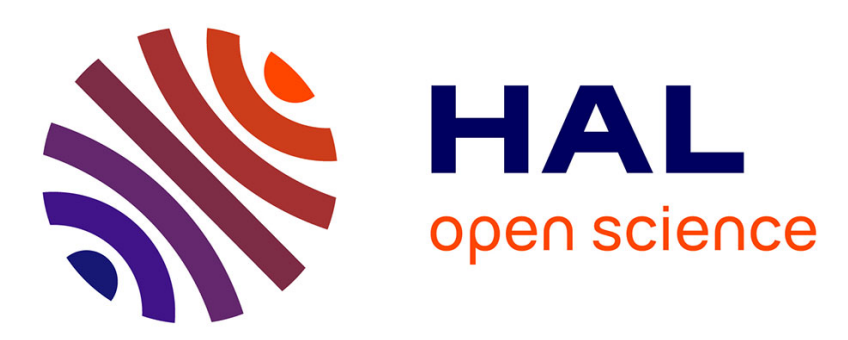

\title{
Closed form solution for the distribution of the total time spent in a subset of states of a homogeneous Markov process during a finite observation period Bruno Sericola
}

\section{- To cite this version:}

Bruno Sericola. Closed form solution for the distribution of the total time spent in a subset of states of a homogeneous Markov process during a finite observation period. [Research Report] RR-0903, INRIA. 1988. inria-00075653

\section{HAL Id: inria-00075653 \\ https://hal.inria.fr/inria-00075653}

Submitted on 24 May 2006

HAL is a multi-disciplinary open access archive for the deposit and dissemination of scientific research documents, whether they are published or not. The documents may come from teaching and research institutions in France or abroad, or from public or private research centers.
L'archive ouverte pluridisciplinaire HAL, est destinée au dépôt et à la diffusion de documents scientifiques de niveau recherche, publiés ou non, émanant des établissements d'enseignement et de recherche français ou étrangers, des laboratoires publics ou privés. 


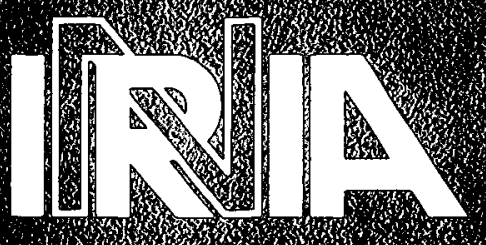

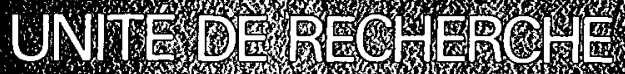
IV) 1 - 3 in:

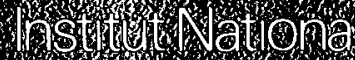

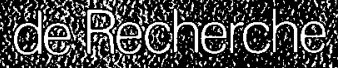

(ำ et r.

Doman- vevolueeau Rocoutencourt - 105

78153 Le chesnay Cedex rance

Tély 39635511

\section{Rapports de Recherche}

$\mathbf{N}^{\circ} 903$

\section{CLOSED FORM SOLUTION FOR} THE DISTRIBUTION OF THE TOTAL TIME SPENT IN A SUBSET OF STATES OF A HOMOGENEOUS MARKOV PROCESS DURING A FINITE OBSERVATION PERIOD

\section{Programme 3}

Bruno SERICOLA 
CLOSED FORM SOLUTION FOR THE DISTRIBUTION OF THE TOTAL TIME SPENT IN A SUBSET OF STATES OF A HOMOGENEOUS MARKOV PROCESS DURING A FINITE OBSERVATION PERIOD

Bruno SERICOLA

Publication Interne $n^{\circ} 424$

Septembre 1988
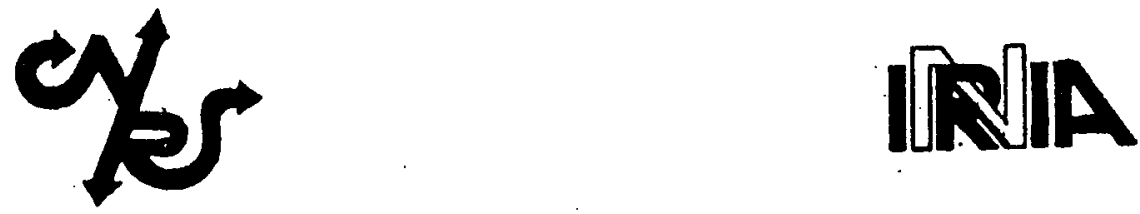
Campus Universitaire de Beaulieu 35042 - RENNES CÉDEX FRANCE

Téléphone: 99362000

Télex: UNIRISA $950473 \mathrm{~F}$

Télécopie: 99383832

\title{
Closed form solution for the distribution of the total time spent in a subset of states of a homogeneous Markov process during a finite observation period
}

\author{
Bruno Sericola \\ Publication Interne $\mathrm{n}^{\circ} 424$ - Septembre 1988 - 20 Pages \\ IRISA Campus de Beaulieu \\ 35042 Rennes Cedex, FRANCE \\ September 5, 1988
}

\begin{abstract}
Markov processes are widely used to modelled computer systems. De Souza e Silva and Gail ([1]) calculated numerically by induction the distribution of cumulative operational time of repairable computer systems, which is the distribution of the total time during which the system was in operation over a finite observation period. An extension of their approach is presented here. We obtain a closed form solution for the distribution of the total time spent in a subset of states of a homogeneous Markov process during a finite observation period ,which is theoretically and numerically interesting. We give also an application of this result to a fault tolerant system.
\end{abstract}

Index terms - Markov processes, uniformization technique, reliability, cumulative operational time, repairable computer systems. 


\title{
Solution explicite pour la distribution du temps total passé dans un sous-ensemble d'états d'un processus markovien homogène durant une période d'observation finie
}

\begin{abstract}
Résumé
Les processus markoviens sont couramment utilisés pour modéliser les systèmes informatiques. De Souza e Silva et Gail ([1]) ont calculé numériquement par récurrence la distribution du temps opérationnel cumulé de systèmes informatiques réparables qui est la distribution du temps total pendant lequel le système est opérationnel sur une période d'observation finie. Une extension de ce résultat est présentée ici. On obtient une expression explicite pour la distribution du temps total passé dans un sous-ensemble d'états d'un processus markovien homogène durant une période d'observation finie, expression intéressante du point de vue théorique et aussi du point de vue numérique. Une application de ce résultat à un système tolérant les pannes est proposée.
\end{abstract}

Mots-clés - Processus markoviens, technique d'uniformisation, fiabilité, temps opérationnel cumulé, systèmes informatiques réparables. 


\section{Introduction}

Consider a continuous-time homogeneous Markov process, say $X=\left(X_{t}\right)_{t \geq 0}$, over a finite state space denoted by $E$. The states of $E$ are divided into two disjoint subsets. In practice, one subset may represent the states for which the system is up (the operational states) and the other the states for which the system is down (the failed states). This division is supposed to be made by some criteria specified by the user. Let $L, 1 \leq L<M$, be the number of operational states, we define:

$$
\begin{aligned}
B & =\{1, \ldots, L\} \quad \text { (set of operational states) } \\
B^{c} & =\{L+1, \ldots, M\} \text { (set of failed states) } \\
E & =\{1, \ldots,, M\}=B \cup B^{c}
\end{aligned}
$$

We suppose that the system, modelled by such a process, is used during a finite interval of time denoted by $(0, t)$. The random variable of interest is the total time spent by the Markov process $X$ into the subset of states $B$ during $(0, t)$. Denoting by $W_{t}$ this random variable, we have:

$$
W_{t}=\int_{0}^{t} \mathbf{1}_{(X, \in B)} d s
$$

where 1 denote the indicator variable. If $B$ is a subset of transient states of $E$, the distribution of $W_{\infty}$ can be found in [2] for semi-Markov reward processes. Assume that the process $X$ is given by its infinitesimal generator $A$ and by its initial probability distribution $\alpha$. Let $A(i, j)$ denote the $(i, j)$ entry of the matrix $A$ and let us define:

$$
\lambda(i)=-A(i, i)=-\sum_{j \neq i} A(i, j)
$$

$\lambda(i)$ represents the output rate from state $i$.

To obtain the distribution of the random variable $W_{t}$, we first recall in the following section the result obtained in [1]; then, in the section 3 , we give the distribution ố the totai numòer of̂ visitis to the states ố a iransient subset for a Markov chain. In section 4 , using the results of the previous sections, we obtain a closed form solution for the distribution of $W_{t}$. Finally, the section 5 gives an application of this result to a fault tolerant system and the section 6 is the conclusion of this paper. 


\section{Distribution of $W_{t}$}

We first recall that the uniformization technique [3] consists of constructing an auxiliary homogeneous Markov chain $Z=\left\{Z_{n}, n \in \mathbb{N}\right\}$ on the state space $E$, with transition probability matrix $P$, and a Poisson process $\{N(t), t \geq 0\}$ independent of $Z$ with rate $\lambda$, such that the two processes $X_{t}$ and $Z_{N(t)}$ are equivalent. The construction is made as follows:

- we choose $\lambda \in \mathbb{R}$ such that: $\lambda \geq \max (\lambda(i), i \in E)$

- we define $P\left(Z_{n+1}=j / Z_{n}=i\right)=P(i, j)=\left\{\begin{array}{cc}1-\frac{\lambda(i)}{\lambda} & \text { if } i=j \\ \frac{A(i, j)}{\lambda} & \text { otherwise }\end{array}\right.$

Between the matrices $A$ and $P$ the following relation holds:

$$
P=I+A / \lambda
$$

where $I$ is the $M \times M$ identity matrix. For every $i, j \in E$, we obtain:

$$
P\left(X_{t}=j / X_{0}=i\right)=\sum_{n=0}^{+\infty} e^{-\lambda t} \frac{(\lambda t)^{n}}{n !} P^{n}(i, j)
$$

Using some known results about the Poisson process, de Souza e Silva and Gail ([1]) show that, for $s<t$, (so that $k \leq n$ ):

$$
P\left[\begin{array}{l|l}
W_{t} \leq s & \begin{array}{l}
\mathrm{n} \text { transitions in }(0, t), \\
\mathrm{k} \text { visits to } B
\end{array}
\end{array}\right]=\sum_{i=k}^{n} C_{n}^{i}\left(\frac{s}{t}\right)^{i}\left(1-\frac{s}{t}\right)^{n-i}
$$

Let $\Omega(n, k), 0 \leq k \leq n+1$, be the probability that the uniformized Markov chain visits $k$ states of $B$ during $n$ transitions. Unconditioning on the number of visits to the states of $B$, we have:

$$
P\left(W_{t} \leq s / n \text { transitions on }(0, t)\right)=\sum_{k=0}^{n} \Omega(n, k) \sum_{i=k}^{n} C_{n}^{i}\left(\frac{s}{t}\right)^{i}\left(1-\frac{s}{t}\right)^{n-i}
$$

Finally, unconditioning on the number of transitions in $(0, t)$, they obtain the following relation (for $s<t$ ): 


$$
P\left(W_{t} \leq s\right)=\sum_{n=0}^{+\infty} e^{-\lambda t} \frac{(\lambda t)^{n}}{n !} \sum_{k=0}^{n} \Omega(n, k) \sum_{i=k}^{n} C_{n}^{i}\left(\frac{s}{t}\right)^{i}\left(1-\frac{s}{t}\right)^{n-i}
$$

For $s \geq t$, it is easy to verify that: $P\left(W_{t} \leq s\right)=1$. In the following, the interest is to give a closed form solution for $\Omega(n, k)$ and so a closed form solution for the distribution of $W_{t}$.

\section{Total number of visits to the states of a transient subset for a Markov chain}

This section is independent of the others and will be used in the following section to give a closed form solution for $\Omega(n, k)$. We consider here a Markov chain with finite state space containing transient states and we give the distribution of the total number of visits to the states of a transient subset which will be denoted by $B$. The recurrent states (eventually absorbing states) can be lumped in only one absorbing state since only the transient states are concerned in this section. So, we will assume that the state space of the Markov chain is composed only with transient states and with one absorbing state.

Let $Z=\left\{Z_{n}, n \in \mathbb{N}\right\}$ a Markov chain on the finite state space $E=\{1, \ldots, m, m+$ $1\}$ where $1, \ldots, m$ are the transient states and where $m+1$ is the absorbing state. Let $B=\{1, \ldots, l\}$, with $1 \leq l \leq m$ and $D=\{l+1, \ldots, m$,$\} when l<m$. With these notations, we have: $E=B \cup D \cup\{m+1\}$. For the conditional probabilities on the initial state, we will use the following notation: $P_{i}()=.P\left(. / X_{0}=i\right)$. We will denote $\alpha=\left(\alpha_{1}, \ldots, \alpha_{l}, \alpha_{l+1}, \ldots, \alpha_{m}, \alpha_{m+1}\right)$ the initial probability distribution of $Z$ and we decompose the first $m$ components in $\alpha_{B}=\left(\alpha_{1}, \ldots, \alpha_{l}\right)$ and $\alpha_{D}=\left(\alpha_{l+1}, \ldots, \alpha_{m}\right)$ when $l<m$. We will denote also $P=(P(i, j)), i, j \in E$ the transition probability matrix of $Z$ and we decompose it with respect to the partition $\{B, D,\{m+1\}\}$ as follows:

$$
P=\left(\begin{array}{ccc}
P_{B} & P_{B D} & P_{B\{m+1\}} \\
P_{D B} & P_{D} & P_{D\{m+1\}} \\
0_{\{m+1\} B} & 0_{\{m+1\} D} & 1
\end{array}\right)
$$

where $0_{\{m+1\} B}$ and $0_{\{m+1\}}$ are row vectors with all entries equal to 0 and with 
respective dimensions $l$ and $m-l$. Let $N_{B}$ be the random variable: "total number of visits of $Z$ to the states of the subset $B^{n}$. That is,

$$
N_{B}=\sum_{i=0}^{+\infty} \mathbf{1}_{\left(X_{i} \in B\right)}
$$

Let us introduce the following notations.

- $\mathbb{I}_{B}$ and $\amalg_{D}$ are column vectors with all entries equal to 1 and with respective dimensions $l$ and $m-l$.

- $I_{B}$ et $I_{D}$ are identity matrices with respective dimensions $l$ and $m-l$.

The following theorem gives the distribution of the random variable $N_{B}$.

\section{Theorem 3.1}

$$
\begin{aligned}
& P\left(N_{B}=0\right)=1-\beta 1_{B} \\
& P\left(N_{B}=k\right)=\beta H^{k-1}\left(I_{B}-H\right) 1_{B} \quad k \geq 1
\end{aligned}
$$

where $\beta, l$ dimension row vector, and $H, l \times l$ matrix, are given by:

$$
\begin{aligned}
\beta & =\alpha_{B}+\alpha_{D}\left(I_{D}-P_{D}\right)^{-1} P_{D B} \\
H & =P_{B}+P_{B D}\left(I_{D}-P_{D}\right)^{-1} P_{D B}
\end{aligned}
$$

Proof. Let us first introduce the two following column vectors:

- $V_{B}(k)=\left(P_{i}\left(N_{B}=k\right)\right), 1 \leq i \leq l, k \geq 0$

- $V_{D}(k)=\left(P_{i}\left(N_{B}=k\right)\right), l+1 \leq i \leq m, k \geq 0$

For every $i \in D$, we have:

$$
\begin{aligned}
P_{i}\left(N_{B}=0\right) & =\sum_{n=0}^{+\infty} P_{i}\left(Z_{1} \in D, \ldots, Z_{n} \in D, Z_{n+1}=m+1\right) \\
& =\sum_{n=0}^{+\infty} \sum_{h=l+1}^{m} P_{i}\left(Z_{1} \in D, \ldots, Z_{n-1} \in D, Z_{n}=h, Z_{n+1}=m+1\right) \\
& =\sum_{n=0}^{+\infty} \sum_{h=l+1}^{m} P_{i}\left(Z_{n+1}=m+1 / Z_{1}, \ldots, Z_{n-1} \in D, Z_{n}=h\right) \\
& =\sum_{n=0}^{+\infty} \sum_{h=l+1}^{m} P\left(Z_{1}, \ldots, Z_{n-1} \in D, Z_{n}=h\right) \\
& =\sum_{n=0}^{+\infty} \sum_{h=l+1}^{m} P_{i}\left(Z_{1}, \ldots, Z_{n-1} \in D, Z_{n}=h\right) P(h, m+1)
\end{aligned}
$$


To continue, we first prove the following lemma.

Lemma 3.2 Let $i$ and $h$ two states of $D$, then for every $n \geq 0$,

$$
P_{i}\left(Z_{1} \in D, \ldots, Z_{n-1} \in D, Z_{n}=h\right)=P_{D}^{n}(i, h)
$$

Proof. The proof is made by induction.

$$
\begin{aligned}
\text { for } n=0, \quad P_{i}\left(Z_{0}=h\right) & =I_{D}(i, h) \\
& =P_{D}^{0}(i, h) \\
\text { for } n=1, \quad P_{i}\left(Z_{1}=h\right) & =P(i, h) \\
& =P_{D}(i, h) \\
\text { for } n=2, \quad P_{i}\left(Z_{1} \in D, Z_{2}=h\right) & =\sum_{r=l+1}^{m} P_{i}\left(Z_{1}=r, Z_{2}=h\right) \\
& =\sum_{r=l+1}^{m} P(i, r) P(r, h) \\
& =P_{D}^{2}(i, h)
\end{aligned}
$$

Suppose the lemma true until the order $n-1$, that is:

$$
P_{i}\left(Z_{1} \in D, \ldots, Z_{n-2} \in D, Z_{n-1}=h\right)=P_{D}^{n-1}(i, h)
$$

We then have:

$$
\begin{aligned}
P_{i}\left(Z_{1}, \ldots, Z_{n-1} \in D, Z_{n}=h\right)= & \sum_{r=l+1}^{m} P_{i}\left(Z_{1}, \ldots, Z_{n-2} \in D, Z_{n-1}=r, Z_{n}=h\right) \\
= & \sum_{r=l+1}^{m} P_{i}\left(Z_{n}=h / Z_{1}, \ldots, Z_{n-2} \in D, Z_{n-1}=r\right) \\
& P_{i}\left(Z_{1}, \ldots, Z_{n-2} \in D, Z_{n-1}=r\right) \\
= & \sum_{r=l+1}^{m} P\left(Z_{n}=h / Z_{n-1}=r\right) \\
& P_{i}\left(Z_{1}, \ldots, Z_{n-2} \in D, Z_{n-1}=r\right) \\
= & \sum_{r=l+1}^{m} P_{D}^{n-1}(i, r) P(r, h) \\
= & P_{D}^{n}(i, h)
\end{aligned}
$$


which ended the proof of the lemma

Thanks to this lemma, we have: for every $i \in D$,

$$
P_{i}\left(N_{B}=0\right)=\sum_{n=0}^{\infty} \sum_{h=l+1}^{m} P_{D}^{n}(i, h) P(h, m+1)
$$

which gives in matrix notation:

$$
V_{D}(0)=\sum_{n=0}^{\infty} P_{D}^{n} P_{D\{m+1\}}
$$

This series converges since $D$ is a transient subset, we then obtain:

$$
V_{D}(0)=\left(I_{D}-P_{D}\right)^{-1} P_{D\{m+1\}}
$$

The matrix $P$ being stochastic, using (1), we have:

$$
\begin{aligned}
& P_{B} \mathbb{1}_{B}+P_{B D} \mathbb{1}_{D}+P_{B\{m+1\}}=\mathbb{\amalg}_{B} \\
& P_{D B} \mathbb{1}_{B}+P_{D} \mathbb{1}_{D}+P_{D\{m+1\}}=\mathbb{I}_{D}
\end{aligned}
$$

This gives easily:

$$
\begin{aligned}
& P_{B\{m+1\}}=\left(I_{B}-P_{B}\right) \amalg_{B}-P_{B D} \amalg_{D} \\
& P_{D\{m+1\}}=\left(I_{D}-P_{D}\right) \mathbb{1}_{D}-P_{D B} \amalg_{B}
\end{aligned}
$$

Replacing now into the expression (2), we obtain:

$$
V_{D}(0)=\mathbb{1}_{D}-\left(I_{D}-P_{D}\right)^{-1} P_{D B} \amalg_{B}
$$

Finally, since

$$
P\left(N_{B}=0\right)=\alpha_{B} V_{B}(0)+\alpha_{D} V_{D}(0)+\alpha_{m+1}
$$

and since $V_{B}(0)$ is equal to 0 , we obtain, thanks to (4):

$$
P\left(N_{B}=0\right)=\alpha_{D} \amalg_{D}-\alpha_{D}\left(I_{D}-P_{D}\right)^{-1} P_{D B} \amalg_{B}+\alpha_{m+1}
$$

The initial probability vector verifying

$$
\alpha_{B} \amalg_{B}+\alpha_{D} \amalg_{D}+\alpha_{m+1}=1
$$


we have:

$$
\begin{aligned}
P\left(N_{B}=0\right) & =1-\left(\alpha_{B}+\alpha_{D}\left(I_{D}-P_{D}\right)^{-1} P_{D B}\right) \amalg_{B} \\
& =1-\beta \Pi_{B}
\end{aligned}
$$

which is the first assertion of the Theorem 3.1.

To prove the second assertion, we write, for $k \geq 1$ and $i \neq m+1$,

$$
P_{i}\left(N_{B}=k\right)=\sum_{j=1}^{m+1} P(i, j) P_{i}\left(N_{B}=k / Z_{1}=j\right)
$$

Taking into account the properties of the Markov chains, we have:

$$
\begin{aligned}
& \forall i \in B, \quad P_{i}\left(N_{B}=k / Z_{1}=j\right)=P_{j}\left(N_{B}=k-1\right) \\
& \forall i \in D, \quad P_{i}\left(N_{B}=k / Z_{1}=j\right)=P_{j}\left(N_{B}=k\right)
\end{aligned}
$$

So,

$$
\begin{aligned}
& \forall i \in B, \quad P_{i}\left(N_{B}=k\right)=\sum_{j=1}^{m+1} P(i, j) P_{j}\left(N_{B}=k-1\right) \quad k \geq 1 \\
& \forall i \in D, \quad P_{i}\left(N_{B}=k\right)=\sum_{j=1}^{m+1} P(i, j) P_{j}\left(N_{B}=k\right) \quad k \geq 1
\end{aligned}
$$

This gives in matrix notation:

$$
\begin{aligned}
& V_{B}(1)=P_{B} V_{B}(0)+P_{B D} V_{D}(0)+P_{B\{m+1\}} \\
& V_{B}(k)=P_{B} V_{B}(k-1)+P_{B D} V_{D}(k-1) \quad k>1 \\
& V_{D}(k)=P_{D B} V_{B}(k)+P_{D} V_{D}(k) \quad k \geq 1
\end{aligned}
$$

Remark that $\forall k \geq \mathrm{i}, P_{m+1}\left(N_{B}=k\right)=0$ and $P_{m+1}\left(N_{B}=0\right)=1$. The relation (8) may be use to write $V_{D}(k)$ in fonction of $V_{B}(k)$ :

$$
V_{D}(k)=\left(I_{D}-P_{D}\right)^{-1} P_{D B} V_{B}(k) \quad k \geq 1
$$

Replacing this value into (7), we obtain:

$$
\begin{aligned}
V_{B}(k) & =\left(P_{B}+P_{B D}\left(I_{D}-P_{D}\right)^{-1} P_{D B}\right) V_{B}(k-1) \quad k>1 \\
& =H V_{B}(k-1) \quad k>1
\end{aligned}
$$


which gives easily:

$$
V_{B}(k)=H^{k-1} V_{B}(1) \quad k>1
$$

To obtain $V_{B}(1)$, we replace $P_{B\{m+1\}}$ and $V_{D}(0)$ by their respective values (3) and (4) into (6). So,

$$
\begin{aligned}
V_{B}(1) & =P_{B D}\left(1_{D}-\left(I_{D}-P_{D}\right)^{-1} P_{D B} \mathbb{1}_{B}\right)+\left(I_{B}-P_{B}\right) \mathbb{1}_{B}-P_{B D} \mathbb{1}_{D} \\
& =\left(I_{B}-P_{B}-P_{B D}\left(I_{D}-P_{D}\right)^{-1} P_{D B}\right) \mathbb{1}_{B} \\
& =\left(I_{B}-H\right) \mathbb{1}_{B}
\end{aligned}
$$

The relation (10) is now the following:

$$
V_{B}(k)=H^{k-1}\left(I_{B}-H\right) 1_{B} \quad k \geq 1
$$

Given that $\forall k \geq 1, P_{m+1}\left(N_{B}=k\right)=0$, we have:

$$
P\left(N_{B}=k\right)=\alpha_{B} V_{B}(k)+\alpha_{D} V_{D}(k) \quad k \geq 1
$$

Replacing $V_{B}(k)$ and $V_{D}(k)$ by their respective values (9) and (11), we conclude the proof:

$$
\begin{aligned}
& P\left(N_{B}=k\right) \\
= & \alpha_{B} H^{k-1}\left(I_{B}-H\right) 1_{B}+\alpha_{D}\left(I_{D}-P_{D}\right)^{-1} P_{D B} H^{k-1}\left(I_{B}-H\right) \amalg_{B} \quad k \geq 1 \\
= & \beta H^{k-1}\left(I_{B}-H\right) \amalg_{B} \quad k \geq 1
\end{aligned}
$$

which is the second assertion of Theorem 3.1.

\section{Closed form solutions for $\Omega(n, k)$ and for the dis- tribution of $W_{t}$}

Recall the notations of the first two sections. $\Omega(n, k), 0 \leq k \leq n+1$, is the probability that the uniformized Markov chain $Z$, defined in section 2, visits $k$ states of $B$ in $n$ transitions. That is,

$$
\Omega(n, k)=P(\mathrm{k} \text { visits to } \mathrm{B} / \mathrm{n} \text { transitions })
$$


Let us decompose the transition probability matrix $P$ and the initial probability vector $\alpha$ of the Markov chain $Z$ with respect to the partition $\left\{B, B^{c}\right\}$ of $E$ as follows:

$$
P=\left(\begin{array}{cc}
P_{B} & P_{B B^{c}} \\
P_{B^{c} B} & P_{B^{c}}
\end{array}\right) \alpha=\left(\alpha_{B}, \alpha_{B^{c}}\right)
$$

For fixed $n,(n \geq 0)$, we construct the following Markov chain which will be denoted by $Z(n)$ and which coresponds to $n$ transitions made on the Markov chain $Z$. We construct the state space $E(n)$ of $Z(n)$ by taking $n+1$ copies of each state of $Z$ plus one absorbing state which will be denoted by $a$. We decompose $E(n)$, which cardinality is $M(n+1)+1$, as follows:

$$
E(n)=B(n) \cup B^{c}(n) \cup\{a\}
$$

with:

$$
B(n)=\bigcup_{i=0}^{n} B_{i} \text { and } B^{c}(n)=\bigcup_{i=0}^{n} B_{i}^{c}
$$

where:

$$
B_{i}=\left\{1_{i}, \ldots, L_{i}\right\} \quad \text { et } \quad B_{i}^{c}=\left\{(L+1)_{i}, \ldots, M_{i}\right\}
$$

To clarify the construction of the transition probability matrix and of the initial probability vector of the markov chain $Z(n)$, we reorder the states of $E(n)$ as follows:

$$
\begin{aligned}
E(n)= & \left\{1_{0}, \ldots, L_{0}, 1_{1}, \ldots, L_{1}, \cdots, 1_{n}, \ldots, L_{n},(L+1)_{0}, \ldots, M_{0}\right. \\
& \left.(L+1)_{1}, \ldots, M_{1}, \cdots,(L+1)_{n}, \ldots, M_{n}, a\right\}
\end{aligned}
$$

The $n+1$ copies of each state are represented by the integer in subscript which goes from 0 to $n$.

We denote by $\alpha(n)$ the initial probability vector of $Z(n)$ which dimension is $M(n+1)+1$. We take:

$$
\alpha(n)=\left(\alpha_{B}(n), \alpha_{B^{c}}(n), \alpha_{a}\right)
$$

where $\alpha_{a}=0$ (i.e. the probability that the chain $Z(n)$ starts in the absorbing state $a$ is equal to 0 ) and: 


$$
\begin{gathered}
\alpha_{B}(n)=\left(\alpha_{B}, 0, \ldots, 0\right) \text { which dimension is } L(n+1) \\
\alpha_{B^{c}}(n)=\left(\alpha_{B^{c}}, 0, \ldots, 0\right) \text { which dimension is }(M-L)(n+1)
\end{gathered}
$$

We denote by $P(n)$ the $(M(n+1)+1, M(n+1)+1)$ transition probability matrix of $Z(n)$ and we take:

for every $i, j \in E$ and for every $x, y \in\{0,1, \ldots, n\}$,

$$
\begin{aligned}
& P(n)\left(i_{x}, j_{y}\right)=\left\{\begin{array}{cc}
P(i, j) & \text { if } y=x+1, x \neq n \\
0 & \text { if } y \neq x+1
\end{array}\right. \\
& P(n)\left(a, j_{x}\right)=0 ; \quad P(n)(a, a)=1 \\
& P(n)\left(i_{x}, a\right)= \begin{cases}1 & \text { if } x=n \\
0 & \text { else }\end{cases}
\end{aligned}
$$

The matrix $P(n)$ so obtained is shown in Figure 1. If we denote now by $\mathbb{1}_{B}$ and by $\mathbb{1}_{B^{\circ}}$ the column vectors with all entries equal to 1 and with respective dimensions $L$ and $M-L$. A block representation for the initial probability vector and for the transition probability matrix $P(n)$ of $Z(n)$ are the following:

$$
\alpha(n)=\left(\begin{array}{cccccc|cccccc|l}
B_{0} & B_{1} & B_{2} & \cdots & B_{n-1} & B_{n} & B_{0}^{c} & B_{1}^{c} & B_{2}^{c} & \cdots & B_{n-1}^{c} & B_{n}^{c} & a \\
\alpha_{B} & 0 & 0 & \cdots & 0 & 0 & \alpha_{B^{c}} & 0 & 0 & \cdots & 0 & 0 & 0
\end{array}\right)
$$

If we denote by $N_{B(n)}$ the random variable: "total number of visits of $Z(n)$ to the states of the subset $B(n)^{n}$, we deduce easily by construction that:

$$
\Omega(n, k)=P\left(N_{B(n)}=k\right) \text { for every } 0 \leq k \leq n+1
$$

Let us now decompose the transition probability matrix $P(n)$ of $Z(n)$ with respect to the partition $\left\{B(n), B^{c}(n),\{a\}\right\}$, we obtain:

$$
P(n)=\left(\begin{array}{ccc}
P_{B}(n) & P_{B^{c}}(n) & P_{B\{a\}}(n) \\
P_{B^{c} B}(n) & P_{B^{c}}(n) & P_{B^{c}\{a\}}(n) \\
0_{\{a\} B}(n) & 0_{\{a\} B^{c}}(n) & 1
\end{array}\right)
$$




\begin{tabular}{|c|c|c|c|c|c|c|c|c|c|c|c|c|c|}
\hline & $B_{0}$ & $B_{1}$ & $B_{2}$ & ... & $B_{n-1}$ & $B_{n}$ & $B_{0}^{c}$ & $B_{1}^{c}$ & $B_{2}^{c}$ & $\cdots$ & $B_{n-1}^{c}$ & $B_{n}^{c}$ & $a$ \\
\hline$B_{0}$ & 0 & $P_{B}$ & 0 & $\cdots$ & 0 & 0 & 0 & $P_{B B^{\circ}}$ & 0 & $\ldots$ & 0 & 0 & 0 \\
\hline$B_{1}$ & 0 & 0 & $P_{B}$ & $\cdots$ & 0 & 0 & 0 & 0 & $P_{B B^{c}}$ & .. & 0 & 0 & 0 \\
\hline$B_{2}$ & 0 & 0 & 0 & $\cdots$ & 0 & 0 & 0 & 0 & 0 & $\ldots$ & 0 & 0 & 0 \\
\hline$\vdots$ & & & & & $\ddots$ & & & & & & $\ddots$ & & $\vdots$ \\
\hline$B_{n-1}$ & 0 & 0 & 0 & ... & 0 & $P_{B}$ & 0 & 0 & 0 & $\ldots$ & 0 & $P_{B B^{c}}$ & 0 \\
\hline$B_{n}$ & 0 & 0 & 0 & $\cdots$ & 0 & 0 & 0 & 0 & 0 & $\cdots$ & 0 & 0 & $\mathbb{1}_{B}$ \\
\hline$B_{0}^{c}$ & 0 & $P_{B^{c} B}$ & 0 & $\cdots$ & 0 & 0 & 0 & $P_{B^{c}}$ & 0 & $\cdots$ & 0 & 0 & 0 \\
\hline$B_{1}^{c}$ & 0 & 0 & $P_{B^{0} B}$ & $\cdots$ & 0 & 0 & 0 & 0 & $P_{B^{c}}$ & $\ldots$ & 0 & 0 & 0 \\
\hline$B_{2}^{c}$ & 0 & 0 & 0 & .. & 0 & 0 & 0 & 0 & 0 & $\cdots$ & 0 & 0 & 0 \\
\hline$\vdots$ & & & & & $\ddots$ & & & & & & $\ddots$ & & $\vdots$ \\
\hline$B_{n-1}^{c}$ & 0 & 0 & 0 & $\cdots$ & 0 & $P_{B^{c} B}$ & 0 & 0 & 0 & $\cdots$ & 0 & $P_{B^{c}}$ & 0 \\
\hline$B_{n}^{c}$ & 0 & 0 & 0 & $\ldots$ & 0 & 0 & 0 & 0 & 0 & $\cdots$ & 0 & 0 & $\mathbb{1}_{B^{c}}$ \\
\hline$a$ & 0 & 0 & 0 & $\cdots$ & 0 & 0 & 0 & 0 & 0 & $\cdots$ & 0 & 0 & 1 \\
\hline
\end{tabular}

Figure 1: $P(n)$, transition probability matrix of $Z(n)$ 
where the new submatrices introduced here are shown in order Figure 1).

We applying the result of the previous section (Theorem 3.1) to the Markov chain $Z(n)$ and we obtain:

$$
\begin{aligned}
& P\left(N_{B(n)}=0\right)=1-\beta(n) \amalg_{B}(n) \\
& P\left(N_{B(n)}=k\right)=\beta(n) H(n)^{k-1}\left(I_{B}(n)-H(n)\right) \amalg_{B}(n) \quad(k \geq 1)
\end{aligned}
$$

with:

$$
\begin{aligned}
& \beta(n)=\alpha_{B}(n)+\alpha_{B^{c}}(n)\left(I_{B^{c}}(n)-P_{B^{c}}(n)\right)^{-1} P_{B^{c} B}(n) \\
& H(n)=P_{B}(n)+P_{B B^{c}}(n)\left(I_{B^{c}}(n)-P_{B^{c}}(n)\right)^{-1} P_{B^{c} B}(n)
\end{aligned}
$$

where:

- $I_{B}(n)$ is the identity matrix which dimension is $L(n+1)$

- $I_{B^{\circ}}(n)$ is the identity matrix which dimension is $(N-L)(n+1)$

- $\mathrm{I}_{B}(n)$ is the column vector with all entries equal to 1 and which dimension is $L(n+1)$

The dimension of the vector $\beta(n)$ and of the matrix $H(n)$ is $L(n+1)$. Defining for $i \geq 0, U_{i}=P_{B B^{c}} P_{B^{c}}^{i} P_{B^{c} B}$ and after some algebraic manipulations, we obtain:

$$
\begin{gathered}
\beta(n)=\left(\alpha_{B}, \alpha_{B^{c}} P_{B^{c} B}, \alpha_{B^{c}} P_{B^{c}} P_{B^{c} B}, \ldots, \alpha_{B^{c}} P_{B^{c}}^{n-1} P_{B^{c} B}\right) \\
H(n)=\left(\begin{array}{ccccccccc}
0 & P_{B} & U_{0} & U_{1} & U_{2} & \cdots & U_{n-3} & U_{n-2} \\
0 & 0 & P_{B} & U_{0} & U_{1} & \cdots & U_{n-4} & U_{n-3} \\
0 & 0 & 0 & P_{B} & U_{0} & \cdots & U_{n-5} & U_{n-4} \\
0 & 0 & 0 & 0 & P_{B} & \cdots & U_{n-6} & U_{n-5} \\
\vdots & & & & & \ddots & \ddots & \vdots \\
0 & 0 & 0 & 0 & 0 & \ddots & P_{B} & U_{0} \\
0 & 0 & 0 & 0 & 0 & \cdots & 0 & P_{B} \\
0 & 0 & 0 & 0 & 0 & \cdots & 0 & 0
\end{array}\right)
\end{gathered}
$$


Remark that the matrix $H(n)$ verify: $H(n)^{n+1}=0$ and $\beta(n) H(n)^{n} 11_{B}(n)=$ $\alpha_{B} P_{B}^{n} 1_{B}$.

Using 4, we obtain directly the following closed form expression for $\Omega(n, k)$ :

$$
\Omega(n, k)=\left\{\begin{array}{cl}
1-\beta(n) \mathbb{1}_{B}(n) & \text { if } k=0 \\
\beta(n) H(n)^{k-1}\left(I_{B}(n)-H(n)\right) \mathbb{1}_{B}(n) & \text { if } 1 \leq k \leq n+1 \\
0 & \text { if } k \geq n+2
\end{array}\right.
$$

We are now able to give a closed form expression for the distribution of the random variable $W_{t}$.

We have seen in section 2 that:

$$
P\left(W_{t} \leq s / n \text { transitions on }(0, t)\right)=\sum_{k=0}^{n} \Omega(n, k) \sum_{i=k}^{n} C_{n}^{i}\left(\frac{s}{t}\right)^{i}\left(1-\frac{s}{t}\right)^{n-i}
$$

This relation can be written as follows:

$$
P\left(W_{t} \leq s / n \text { transitions on }(0, t)\right)=\sum_{i=0}^{n} C_{n}^{i}\left(\frac{s}{t}\right)^{i}\left(1-\frac{s}{t}\right)^{n-i} \sum_{k=0}^{i} \Omega(n, k)
$$

and:

$$
\begin{gathered}
\sum_{k=0}^{i} \Omega(n, k)=1-\beta(n) \mathbb{I}_{B}(n)+\sum_{k=1}^{i} \beta(n) H(n)^{k-1}\left(I_{B}(n)-H(n)\right) \mathbb{I}_{B}(n) \\
=1-\beta(n) H(n)^{i} \mathbf{I}_{B}(n)
\end{gathered}
$$

So,

$$
\begin{aligned}
& P\left(W_{t} \leq s / n \text { transitions on }(0, t)\right) \\
= & \sum_{i=0}^{n} C_{n}^{i}\left(\frac{s}{t}\right)^{i}\left(1-\frac{s}{t}\right)^{n-i}\left[1-\beta(n) H(n)^{i} \Pi_{B}(n)\right] \\
= & 1-\beta(n) \sum_{i=0}^{n} C_{n}^{i}\left(\frac{s}{t} H(n)\right)^{i}\left(1-\frac{s}{t}\right)^{n-i p} \amalg_{B}(n) \\
= & 1-\beta(n)\left[\frac{s}{t} H(n)+\left(1-\frac{s}{t}\right) I_{B}(n)\right]^{n} \amalg_{B}(n)
\end{aligned}
$$




$$
=1-\beta(n)\left[I_{B}(n)-\frac{s}{t}\left(I_{B}(n)-H(n)\right)\right]^{n} \mathbb{I}_{B}(n)
$$

Finally, for $s<t$, we obtain the following result:

$$
P\left(W_{t} \leq s\right)=1-\sum_{n=0}^{+\infty} e^{-\lambda t} \frac{(\lambda t)^{n}}{n !} \beta(n)\left[I_{B}(n)-\frac{s}{t}\left(I_{B}(n)-H(n)\right)\right]^{n} \mathbb{I}_{B}(n)
$$

which can be written also:

$$
P\left(W_{t} \leq s\right)=1-\sum_{n=0}^{+\infty} e^{-\lambda t} \frac{(\lambda t)^{n}}{n !} \sum_{i=0}^{n} C_{n}^{i}\left(\frac{s}{t}\right)^{i}\left(1-\frac{s}{t}\right)^{n-i} \beta(n) H(n)^{i} \mathrm{I}_{B}(n)
$$

The reliabilty $R(t)$ being defined by $R(t)=P\left(W_{t}=t\right)=1-P\left(W_{t}<t\right)$, we obtain it letting $s$ tend to $t$ in the previous expressions of $P\left(W_{t} \leq s\right)$. So,

$$
\begin{aligned}
R(t) & =P\left(W_{t}=t\right) \\
& =1-P\left(W_{t}<t\right) \\
& =\sum_{n=0}^{+\infty} e^{-\lambda t} \frac{(\lambda t)^{n}}{n !} \beta(n) H(n)^{n} \amalg_{B}(n) \\
& =\sum_{n=0}^{+\infty} e^{-\lambda t} \frac{(\lambda t)^{n}}{n !} \alpha_{B} P_{B}^{n} 1_{B} \\
& =\alpha_{B} e^{A_{B} t} 1_{B}
\end{aligned}
$$

where $A_{B}$ is the $(L, L)$ matrix deduced from $A$ (see section 1 ) by deleting the rows and columns corresponding to the states of $B^{c}$. That is, $\forall i, j \in B, A_{B}(i, j)=A(i, j)$.

\section{Application to a fault tolerant system}

Consider a system with 3 identical and independent components. Only one of these components is performing tasks. Each component fails with a constant failure rate $\phi$. When the performing component fails, a recovery process try to reconfigure the system replacing the failed component by the following. The expected time to execute the recovery process is equal to $1 / \rho$. The success probability of the recovery process is constant $d$ (the unsuccess means the crash of the system). Note that the recovery 


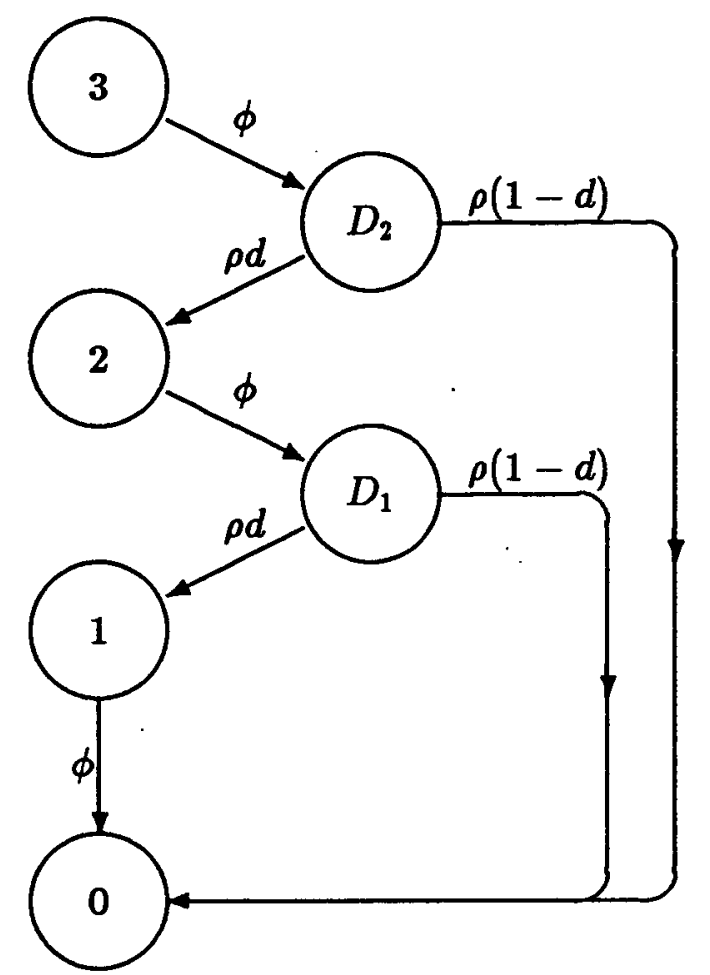

Figure 2: A fault tolerant 3-components system

rate $\rho$ is more larger than the failure rate $\phi$. The Markov process associated to this system is shown in Figure 2. The states $D_{1}, D_{2}$ are the recovery states and the state 0 is the down state (in these states the system is not operationnal). The states 1, 2, 3 are the operationnal states. The initial state is the state 2 with probability 1.

So, we obtain: $B=\{3,2,1\}$ and $B^{c}=\left\{D_{2}, D_{1}, 0\right\}$. The initial probability distribution is: $\alpha_{B}=\{1,0,0\}$ and $\alpha_{B^{c}}=\{0,0,0\}$. The transition rate matrix $A$ of the obtained Markov process can be easily built thanks to the Figure 2. The quantity of interest is the distribution of the total time spent in the subset $B$ during the interval of time $(0, t)$ which is also distribution of cumulative operationnal time during the utilization period $(0, t)$ of the system.

Due to the above assumptions, we choose: $\lambda=\max (\lambda(i), i \in E)=\rho$. The transition probability matrix $\boldsymbol{P}$ of the uniformized Markov chain has the following properties:

$$
\forall i \geq 1, \quad U_{i}=P_{B B^{c}} P_{B^{c}}^{i} P_{B^{c} B}=0
$$


Recall that $U_{0}=P_{B B^{c}} P_{B^{c} B}$. Using these properties, we obtain for every $n \in \mathbb{N}$ :

$$
\beta(n) H(n)^{i} \amalg_{B}(n)=\left\{\begin{array}{lr}
\alpha_{B}\left(P_{B}+U_{0}\right)^{i} 1_{B} & i=0,1, \ldots, n-2 \\
\alpha_{B}\left(P_{B}^{n-1}+(n-1) P_{B}^{n-2} U_{0}\right) \amalg_{B} & i=n-1 \\
\alpha_{B} P_{B}^{n} 1_{B} & i=n
\end{array}\right.
$$

This gives, using the relation (12) of the previous section and after some algebraic manipulation:

$$
\begin{aligned}
P\left(W_{t} \leq s\right)= & 1-\alpha_{B}\left(e^{-\rho s}-\rho(t-s) e^{-\rho t}-e^{-\rho t}\right) e^{\rho s\left(P_{B}+U_{0}\right)} \mathbb{I}_{B} \\
& -\alpha_{B}\left(\rho(t-s) I_{B}+\rho^{2} s(t-s) U_{0}+I_{B}\right) e^{-\rho t} e^{-\rho s P_{B}} \mathbb{I}_{B}
\end{aligned}
$$

If we decompose now the transition rate matrix $A$, with respect to the partition $\left\{B, B^{c}\right\}$, as follows:

$$
A=\left(\begin{array}{cc}
A_{B} & A_{B B^{c}} \\
A_{B^{c} B} & A_{B^{c}}
\end{array}\right)
$$

we obtain the following relation:

$$
\begin{aligned}
P\left(W_{t} \leq s\right)= & 1-\left(1-e^{-\rho(t-s)}-\rho(t-s) e^{-\rho(t-s)}\right) \alpha_{B} e^{\left(A_{B}+A_{\left.B^{c} A^{c} A_{B} c_{B} / \rho\right) s} 1_{B}\right.} \\
& -\alpha_{B}\left(\rho(t-s) I_{B}+s(t-s) A_{B B^{c} A_{B^{c} B}}+I_{B}\right) e^{-\rho(t-s)} e^{A_{B} s} 1_{B}
\end{aligned}
$$

and this gives finally the following relation:

$P\left(W_{t} \leq s\right)=1-e^{-\phi s}\left[1+\left(1-e^{-\rho(t-s)}\right) \phi d s+\left(1-e^{-\rho(t-s)}-\rho(t-s) e^{-\rho(t-s)}\right) \frac{\phi^{2} d^{2} s^{2}}{2}\right]$

\section{Conclusion}

The main contributions of this paper are the closed form solutions given for three probability distributions concerning homogeneous Markov processes. The first is the distribution of the total number of visits to the states of a transient subset for a Markov chain. The second is the distribution of the total number of visits to the states of a subset during the $n^{\text {th }}$ first transition for a Markov chain. The third is the distribution of the total time spent in a subset of states of a Markov process. The principal idea of this paper is to obtain quantities over a finite interval of time thanks to corresponding quantities over an infinite interval of time. 


\section{References}

[1] E. de Souza e Silva and H. R. Gail. Calculating cumulative operational time distributions of repairable computer systems. IEEE Transactions on Computers, C.35:322-332, April 1986.

[2] G. Ciardo, R. Marie, B. Sericola, and K. Trivedi. Performability analysis using semi-Markov process. Technical Report CS-1988-9, Duke Computer Science Technical Report, 1988.

[3] S. M. Ross. Stochastic Processes. John Wiley and Sons, 1983. 
PI 416 - CONTROLE D'EXECUTION EN ROBOTIQUE DE COOPERATION Catherine JAUFFRINEAU

52 Pages, Juin 1988.

PI 417 - UN SCHEMA (ABSTRAIT) D'ITERATION REPARTIE. APPLICATION AU CALCUL DES CHEMINS DE VALEURS MINIMALES

Jean-Michel HELARY, Michel RAYNAL

24 Pages, Juin 1988.

PI 418 - STEREOMETRIE INTERACTIVE POUR LA TELEOPERATION ASSISTEE PAR ORDINATEUR

Alain VERDIER

52 Pages, Juin 1988.

PI 419 - PARTAGE D'OBJETS DANS LES SYSTEMES DISTRIBUES. PRINCIPES DES RAMASSE-MIETTES

André COUVERT, Aomar MADDI, René PEDRONO

60 Pages, Juin 1988.

PI 420 - NONCONFORMING FINITE ELEMENTS FOR THE STOKES PROBLEM Michel CROUZEIX, Richard S. FALK

26 Pages, Juillet 1988.

PI 421 - COMPLING TEMPORAL LOGIC SPECIFICATIONS INTO OBSERVERS Omar DRISSI-KAITOUNI, Claude JARD

18 Pages, Juillet 1988.

PI 422 - DISTANCE MEASURES FOR SIGNAL PROCESSING AND PATTERN RECOGNITION

Michèle BASSEVILLE

50 Pages, Septembre 1988.

PI 423 - MULTIPLE CORRESPONDENCE ANALYSIS WITH MISSING DATA Peter G.M. van der HEIDJEN, Brigitte ESCOFIER

30 Pages, Septembre 1988.

PI 424 - CLOSED FORM SOLUTION FOR THE DISTRIBUTION OF THE TOTAL TIME SPENT IN A SUBSET OF STATES OF A HOMOGENEOUS MARKOV PROCESS DURING A FINITE OBSERVATION PERIOD Bruno SERICOLA

20 Pages, Septembre 1988. 
